\title{
Resolution of a Severe Grade of Obstructive Sleep Apnea Syndrome with Mandibular Advancement Device: A Case Report
}

\author{
Alberto De Stefani, DDS, Giovanni Bruno, DDS, Ludovica Agostini, \\ Luca Mezzofranco, DDS, Antonio Gracco, DDS \\ Department of Neuroscience, University of Padova, Padova, Italy
}

Obstructive sleep apnea syndrome (OSAS) is a disorder of the sleep with episodes of the obstruction at the upper airway that can be recurrent, partial or complete. The continuous positive airway pressure (CPAP) is the gold treatment for OSAS, but many patients do not tolerate this kind of treatment, and for this reason mandibular advancement device are used. A 55-year-old Caucasian man affected by severe OSAS [apnea-hypopnea index (AHI): 51, lowest saturation value (NADIR): 77\%] came at Padua University Hospital Dental Clinic after ENT evaluation to consider an alternative treatment to CPAP. After a surgery treatment and the use of oral appliance (Somnodent, Somnomed Limited) associated with vertical elastics AHI improve to 2.4 and NADIR to $88 \%$.

Sleep Med Res 2020;11(1):44-48

Key Words OSAS, Orthodontics, MAD.

\footnotetext{
Received: January 31, 2020

Revised: April 3, 2020

Accepted: April 14, 2020

Correspondence

Alberto De Stefani, DDS

Department of Neuroscience,

University of Padova, Via Giustiniani,

2-35100 Padova, Italy

Tel +393343345840

E-mail alberto.de.stefani@hotmail.it

ORCID

Alberto De Stefani

https://orcid.org/0000-0001-9087-3953

(c) This is an Open Access article distributed under the terms of the Creative Commons Attribution Non-Commercial License (https://creativecommons.org/licenses/by-nc/4.0) which permits unrestricted non-commercial use, distribution, and reproduction in any medium, provided the original work is properly cited.
}

\section{INTRODUCTION}

Obstructive sleep apnea syndrome (OSAS) is a disorder of the sleep with episodes of the obstruction at the upper airway that can be recurrent, partial or complete [1]. The gold standard test for the diagnosis of OSAS is a polysomnography (PSG) registration or a polygraph registration without electroencephalography.

The severity of OSA is defined with the apnea-hypopnea index (AHI). It consists in the number of mixed obstructive and central episodes of apneas or hypopnease per hour of total sleep time. Three stages of OSA severity are considered: mild when AHI is lower than 15 events/h of sleep; moderate when AHI > 15 and < 30 events/h; and severe when AHI is higher than 30 events/h [2]. The diagnostic criteria for the OSAS are 1) AHI > 5 episodes every hour of sleep, 2) excessive daytime sleepiness, or 3) at least two other symptoms from the following: sleep suffocation, arousal, sleep providing no relaxation, daily fatigue, and daytime attention lapses. There are important effects of daytime activity on the patient, such as humor, productivity and an increased risk of car accidents.

The OSAS treatment can be surgical and non-surgical. The first choice includes nasal, oral/ palate or hypopharyngeal surgery and orthognathic surgery. Non-surgical treatment includes continuous positive airway pressure (CPAP), positional therapy, mandibular advancement devices (MAD) and pharmacological therapy [3]. CPAP is the gold standard non-surgical treatment for OSAS but it has a low compliance in a high percentage of patients. The adherence at the CPAP is reported between $46 \%$ to $83 \%$ [4].

The alternative treatment in patient with mild or moderate condition or in severe patient with no compliance with the CPAP are the MAD. This appliances show and higher compliance than CPAP and adherence and satisfaction were satisfactory: a 5 years study about adherence to MAD demonstrate that $55.8 \%$ of the patients used the MAD regularly [5]. More- 
over, MADs permit to regulate and modified the mandibular position during the treatment in order to achieve gradually the required effects [6]. On the other side, MAD side effect should be considered during the treatment: increasing saliva secretion or dry mouth, tooth or gum discomfort, muscle tenderness, abnormal morning occlusion and a reduction of overjet and overbite [6-8].

The aim of this case report is the description of a case of severe OSAS improved in mild OSAS after surgery and the use of an oral appliance (OA) device.

\section{DIAGNOSIS AND ETIOLOGY}

A 55-year-old Caucasian man affected by severe OSAS came at Padua University Hospital Dental Clinic after ENT evaluation to consider an alternative treatment to CPAP the patient gave the consent that his photo and medical data can be used as a case report.

At the medical history the patient referred arterial hypertension without pharmacological control. He referred snoring and apnea episodes during the night reported by his wife and daytime sleepiness (Epworth Scale Score resulted 14). The patient also reports a feeling of tiredness in the morning, even if he sleeps 7 hours a night, and reports nocturia.
From an occlusal point of view the patient showed an angle first molar and canine class without crowding on both dental arches (Fig. 1). The maximum protrusion was $+6 \mathrm{~mm}$ and maximum retrusion was $-8 \mathrm{~mm}$ resulting in a functional mandibular range of $14 \mathrm{~mm}$, and rest position was $-6 \mathrm{~mm}$. Moreover, in OSAS patients an important parameter is the neck circumference (considerate pathological over $43 \mathrm{~cm}$ for man and $41 \mathrm{~cm}$ for woman) and in this patient was calculated and resulted $41 \mathrm{~cm}$.

Another important risk factor in OSAS patients is the body mass index (defined as the relationship between weight and height) value and and in this patient was 24 .

We also evaluated the upper airway with the use of Cone beam CT (CBCT) (Fig. 2) and the intraoral clinical evaluation of the patient showed a tonsillar I grade and a tongue III level (Mallampati). The patient's chewing muscles were assessed on first visit by palpation, to check that there were no contractures and the temporomandibular joint was assessed through CBCT to exclude signs of resorption of the condylar cortex.

\section{REPORT OF CASE}

As all the OSAS patient the first exam that reveal the diagnosis of OSAS was a polysomnography performed in 2015. In
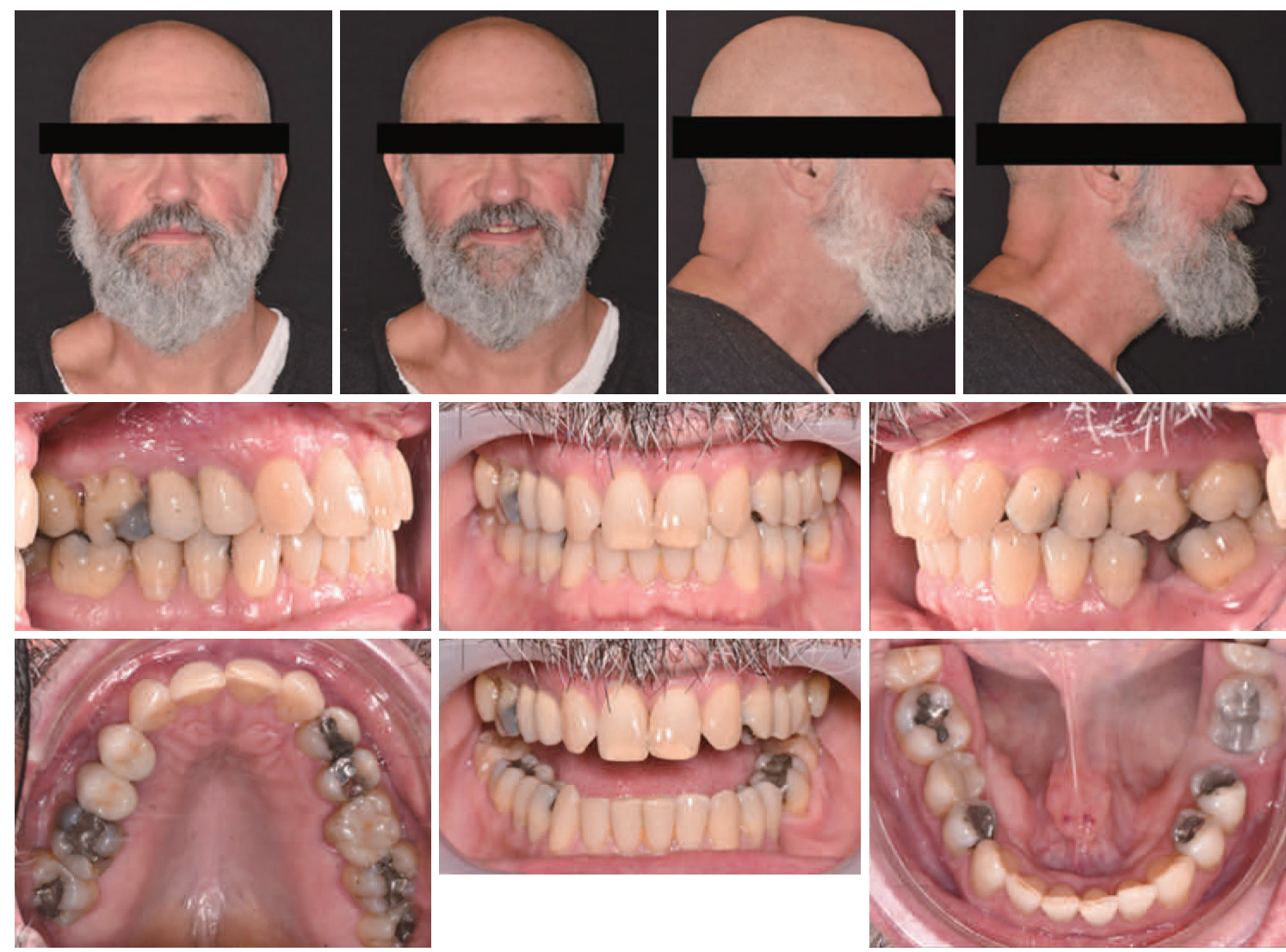

Fig. 1. Intraoral and extraoral pre-treatment photographs. 

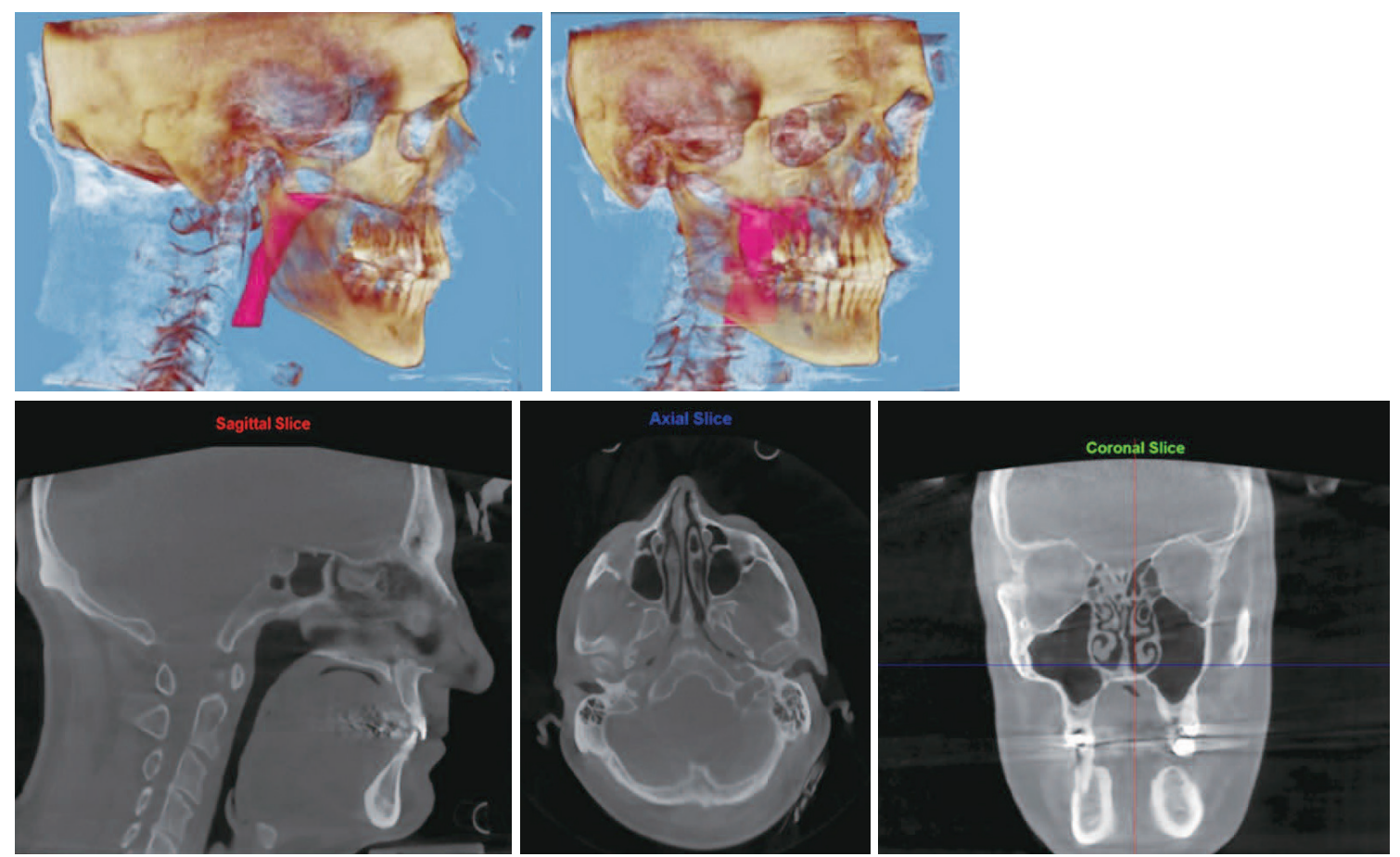

Fig. 2. Pre-treatment cone beam CT of the patient.
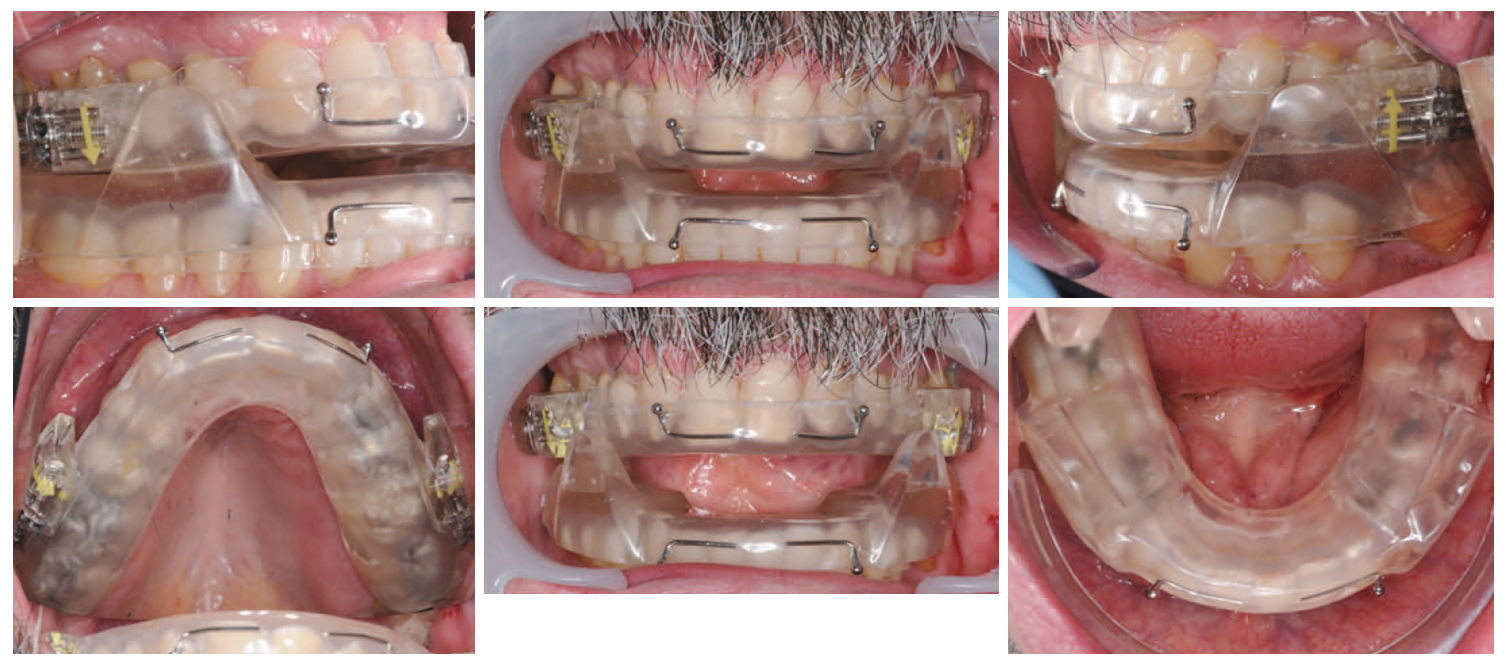

Fig. 3. Intraoral photograph after treatment.

this first polysomnography, performed with a polygraph supplied at home (III level PSG) and reported by a neurologist specialized in sleep medicine, he results to be a severe OSAS: AHI was 51.2. Oxygen average saturation was $91.7 \%$, oxygen desaturation index (ODI) was 43.4 and lowest saturation value (NADIR) was $77 \%$. The AHI in supine position was 63.9 , so the patient was not considerate a positional OSAS.

This polysomnography was reported by a neurologist specialized in sleep medicine. CT90 is value that considerate the time spend with a desaturation under $90 \%$ and in this patient, it was $20 \%$. After this first evaluation the patient was subject to a videolaringoscopy from which it resulted a nasal septum de- viation, hypertrophy of the inferior turbinates and the presence of a neoformation on the right tonsillar pillar.

In 2017 the patient underwent a drug induced sleep endoscopy (DISE) which evidenced an IV degree circular collapse in velopharynx level and also IV degree circular collapse in hypopharynx and oropharynx. During DISE the patient underwent also a septoplasty, inferior turbinate surgery and the excision of the neoformation on the tonsillar pillar. After the histological examination the neoformation was classified as a squamous cell papilloma. The outcome of the surgery was regular.

The second polysomnography (I level) revealed 38.5 AHI score, oxygen average saturation $93.9 \%$ and $78.4 \%$ NADIR, 
shows a great improvement after surgery. ENT suggested to the patient the use of CPAP and an orthodontic evaluation for an OA. At the first dental evaluation the patient reported his choice not to use CPAP, for this reason was programmed an OA MAD called "Somnodent" (Somnomed Limited, Australia) associated with vertical elastics. After this first evaluation dental impressions were taken and also the construction bite, the first protrusion chosen for the patient was $70 \%$ of his total functional mandibular range, considerate a therapeutic position [6]. The maximum protrusive, maximum retrusive and normal position measurements are carried out using an instrument called George Gage (Grate Lake, NY, USA) and equipped with a millimeter ruler.

One month later the appliance was delivered to the patient, and the patient was instructed to side effects of this therapy like increasing saliva secretion or dry mouth in the first time, tooth or mouth discomfort and muscle aching and for this reason the patient was instruct to do mouth and facial muscle stretching every morning (Fig. 3). Muscle stretching exercises include mouth opening and closing and lateral exercises with the use of a hand used as a source of resistance to movement.

\section{RESULTS}

After delivery of the device, the titration phase followed, for which 35 activations were prescribed to the patient. These activations produce an advancement of the device of $0.1 \mathrm{~mm}$ each and are performed by the patient at home every 3 days approximately. Three month later the patients revealed an improvement of symptoms as a reduction of the sleepines during the day and a reduction in snoring. Four months after the delivery of MAD the patients made a third polysomnography (I level) and carried out the sleep recording with the OA: AHI score was 2.4 , oxygen average saturation was $94.2 \%$ and NADIR was $88 \%$.

\section{DISCUSSION}

After the surgery treatment the AHI score is improved from 51.2 to 38.5 and the patient was classified as a severe grade of OSAS even if the score is halved. The use of OA improved AHI value from 38.5 to 2.4 and the patient now is not classified as an OSAS patient. The role of the surgery is really important in treatment of OSAS patient because improvement of upper airway patency. In this case not only the AHI value gets better but also ODI value (from 43.4 to 32.7 ) and the average of saturation (from $91.7 \%$ to $93.9 \%$ ). In this case report MAD give a resolution of a severe case of OSAS and the improvement of AHI value from 38.5 to 2.4. But also the saturation values are better after MAD use: ODI value from $32.7 / \mathrm{h}$ to $1.8 / \mathrm{h}$, NADIR from $78.4 \%$ to $88.0 \%$ and saturation average from $93.9 \%$ to $94.2 \%$
Table 1. PSG at the beginning of the treatment, two years after the videolaringoscopy and four months after the mandibular advancement devices

\begin{tabular}{lccc}
\hline & PSG & PSG & PSG \\
& $05 / 03 / 2015$ & $18 / 01 / 2018$ & $04 / 03 / 2019$ \\
\hline AHI & 51.2 & 38.5 & 2.4 \\
ODI (/h) & 43.4 & 32.7 & 1.8 \\
Oxigen average & 91.7 & 93.9 & 94.2 \\
saturation (\%) & & 78.4 & 88.0 \\
NADIR (\%) & 77.0 & & \\
\hline
\end{tabular}

PSG: polysomnography, AHI: apnea-hypopnea index, ODI: oxygen desaturation index, NADIR: lowest saturation value.

(Table 1).

Such as literature suggest, MAD can be considerate a good alternative for resolution of OSAS for patients who cannot tolerate the use of CPAP. The case report of this study does not tolerate the use of the mask overnight, and the alternative of using OA to reduce the OSA is successful. In fact, the patient was immediately very compliant for this kind of therapy.

This study wants to show that the effectiveness of the therapy with OA should be compared with the use of CPAP, at the moment considerate the gold standard therapy. Moreover Marklund et al. suggest that mandibular advancement devices show a lower efficiency on severe cases with respect to the milder and the reason is unknow, maybe because patients with severe OSAS have large obstruction from the soft palate to the tongue base or epiglottis than patients with mild OSAS [9-13]. This study shows a resolution of a severe case of OSAS with oral device.

\section{CONCLUSION}

This report discuss the importance of a multidisciplinary approach in OSAS patient: ENT evaluation and therapy associated with an dental approach with the use of MAD are primary in the therapeutic pathway of patient affected by OSAS, in particular when the patient not tolerate the use of CPAP. OAs are able to solve a severe grade of OSAS.

\section{Acknowledgments}

None.

\section{Conflicts of Interest}

The authors have no financial conflicts of interest.

\section{Authors' Contribution}

All the authors contributed in all the part of the manuscript.

\section{REFERENCES}

1. Schütz TC, Cunha TC, Moura-Guimaraes T, Luz GP, Ackel-D’Elia C, Alves Eda S, et al. Comparison of the effects of continuous positive airway pressure, oral appliance and exercise training in obstructive sleep apnea syndrome. Clinics (Sao Paulo) 2013;68:1168-74. 
2. Wojda M, Kostrzewa-Janicka J, Bieleń P, Jurkowski P, MierzwińskaNastalska E. Therapeutic efficacy of mandibular advancement devices in patients with severe sleep apnea: a preliminary report. Adv Exp Med Biol 2018;1040:39-46.

3. Verse T, Hörmann K. The surgical treatment of sleep-related upper airway obstruction. Dtsch Arztebl Int 2011;108:216-21.

4. Weaver TE, Grunstein RR. Adherence to continuous positive airway pressure therapy: the challenge to effective treatment. Proc Am Thorac Soc 2008;5:173-8.

5. Tison C, Sébille-Elhage S, Ferri J. [Mandibular advancement device: a 5 -year long experience in obstructive sleep apnea/hypopnea syndrome]. Rev Stomatol Chir Maxillofac 2011;112:80-6.

6. Marklund M, Verbraecken J, Randerath W. Non-CPAP therapies in obstructive sleep apnoea: mandibular advancement device therapy. Eur Respir J 2012;39:1241-7.

7. Tingting X, Danming Y, Xin C. Non-surgical treatment of obstructive sleep apnea syndrome. Eur Arch Otorhinolaryngol 2018;275:335-46.

8. De Stefani A, Bruno G, Mezzofranco L, Perri A, Marchese Ragona R, Gracco A. Multidisciplinary ENT-orthodontic treatment in a hyper- tensive patient affected by severe OSAS. ORAL \& Implantology 2018;6: 59-63.

9. Gracco A, Bruno G, de Stefani A, Ragona RM, Mazzoleni S, Stellini E. Combined orthodontic and surgical treatment in a 8-years-old patient affected by severe obstructive sleep apnea: a case-report. J Clin Pediatr Dent 2018;42:79-84.

10. Marklund M, Franklin KA, Sahlin C, Lundgren R. The effect of a mandibular advancement device on apneas and sleep in patients with obstructive sleep apnea. Chest 1998;113:707-13.

11. Bignotti D, De Stefani A, Mezzofranco L, Bruno G, Gracco A. Multidisciplinary approach in a 12-year-old patient affected by severe obstructive sleep apnea: a case-report. Sleep Med Res 2019;10:103-7.

12. Bruno G, De Stefani A, Conte E, Caragiuli M, Mandolini M, Landi D, et al. A procedure for analyzing mandible roto-translation induced by mandibular advancement devices. Materials (Basel) 2020;13:1826.

13. Crivellin G, Bruno G, De Stefani A, Mazzoli A, Mandolini M, Brunzini A, et al. Strength distribution on TMJ using mandibular advancement device for OSAS treatment: a finite element study. Dental Cadmos 2018; 86:757-64. 